\title{
A smoothed variable horizon peridynamics and its application to the fracture parameters evaluation
}

\author{
M. Imachi · T. Takei · M. Ozdemir • \\ S. Tanaka - S. Oterkus · E. Oterkus
}

Received: / Accepted:

\begin{abstract}
Peridynamics has attractive features for solving several fracture mechanics problems. On the other hand, to ensure the accuracy, a great deal of particles (material points) is required. Introduction of variable horizon is an alternative approach by changing the horizon size over the problem domain. In the present study, we propose a novel variable horizon concept. It is known that undesired "ghost" force arises along the transition region of different horizons. In order to suppress the ghost force, the gradual variation of the horizon size over a certain region, called smoothing length, is introduced between the different scale particle distributions. Efficiency of the smoothed variable horizon peridynamics is demonstrated through several numerical studies employing the ordinary statebased peridynamics. As a basic case, a linear displacement field is considered. It is
\end{abstract}

M. Imachi, T. Takei, S. Tanaka

Graduate School of Advanced Science and Engineering, Hiroshima University, 4-1, Kagamiyama 1-chome, Higashi-Hiroshima,739-8527, Japan

Tel.: +81-82-424-7859

Fax: +81-82-422-8527

E-mail: satoyuki@hiroshima-u.ac.jp

M.Imachi

E-mail: michiya@hiroshima-u.ac.jp

Present address: TechnoStar Co., Ltd, 7-1-1, Akasaka, Minato-ku, Tokyo 107-0052 Japan.

T.Takei

E-mail: takaaki.takei.395@ctc-g.co.jp

Present address: ITOCHU Techno-Solutions Corporation (CTC), North Gate Bldg., 3-1-3, Umeda, Kita-ku, Osaka-shi, Osaka 530-0001 Japan.

M. Ozdemir

Department of Naval Architecture and Marine Engineering, Ordu University, Ordu, Turkey

E-mail: muratozdemir@odu.edu.tr

S. Oterkus, E. Oterkus

Department of Naval Architecture, Ocean and Marine Engineering,

University of Strathclyde, Glasgow, United Kingdom

E-mail: selda.oterkus@strath.ac.uk

E-mail: erkan.oterkus@strath.ac.uk 
observed that the proposed approach significantly reduces the ghost forces along the interface of different spatial discretizations. Additionally, the dynamic stress intensity factors of stationary cracks are carefully examined. The path independence of the fracture parameters in the variable horizon peridynamics is ensured. Efficiency of the presented method is then discussed. It is monitored that introduction of the smoothing length concept significantly reduces the computational costs in the peridynamic modeling.

Keywords Peridynamics · Variable Horizon - Ghost Force · Dynamic Stress Intensity Factor

\section{Introduction}

Peridynamics (PD) is a new continuum mechanics formulation that has been popular in recent years. PD was proposed as a non-local reformulation of classical elasticity equations by Silling [1]. The equation of motion in PD is an integrodifferential equation inherently avoiding the partial differentiations, which can not be evaluated for discontinuities in a traditional manner. In the PD framework, cracks are taken into account in a straightforward way by simply eliminating the interactions between the particles on each side of the crack segment.

Considering brittle fracture phenomenon, various studies including prototype microelastic brittle model have been proposed and its effectiveness has been shown by Ha and Bobaru [2]. Furthermore, the fracture mechanics parameters for stationary cracks have been evaluated by the authors [3,4], and the initiation of dynamic crack propagation and arrest phenomenon have been studied for calculating the stress intensity factors (SIFs) with high accuracy by introducing a transition bond concept $[5,6]$. The ordinary state-based formulation was slightly modified and employed for evaluating crack patterns in functionally graded materials [7].

In the PD formulation, the fracture phenomenon is accounted for whether the interactions (force state) between the particles exist or not. Fracture analysis can be performed without re-meshing unlike FEM, which can be regarded as the superiority of the PD modeling. However, in order to meet sufficient accuracy in the computation, a large number of particles is required and it is necessary to employ a large scale model. Accordingly, in terms of computational cost, much more analysis time is demanded compared to other numerical methods. To overcome this issue, increasing the particle density and modifying the horizon size only in the region, where good accuracy is demanded, are conceivable practices. However, the standard PD formulation was constructed for constant horizon size of all particles, varying the particle arrangement and horizon sizes throughout the problem domain therefore generates undesired forces and spurious wave reflections [8].

To address this issue, the variable horizon PD (VH-PD) concept was proposed by Silling et al. [8]. Dipasquale et al. [9] analyzed crack propagation with 2D PD employing an adaptive grid refinement. Several strategies for avoiding the wave reflection were presented in Ref. [9]. Song et al. [10] also employed the adaptive grid refinement in PD modeling of ice craters under impact loading. The ghost force effect however has not been discussed in detail by Ref. [10]. Additionally, the Dual Horizon PD (DH-PD) formulation dealing with the variable horizons have been presented in [11,12]. Ren et al. [13] implemented DH-PD for the ductile fracture problems employing the non-ordinary state-based PD formulation. Rabczuk 
and Ren [14] also applied DH-PD formulation to address contact and fracture of rock-like materials. Jenabidehkordi and Rabczuk [16] proposed multi-horizon PD model to avoid ghost force effects in case of different horizon sizes. Multiple horizons, which do not coincide each other, are assigned for the particles in the refinement zone. Recently, multiscale coupling model of molecular dynamics and PD was proposed by Tong and $\mathrm{Li}$ [15]. The micro (atomistic) scale is modeled by molecular dynamics and macroscale is modeled by state-based PD. A transition region with adaptive multiscale elements is defined for transmitting physical quantities, e.g., displacements. A filter is implemented in the transition region so that the high frequency instant waves are filtered and a smooth low frequency wave is transmitted from microscale to macroscale. Coupling of PD and FEM was studied by the variable horizon concept in [17]. A coupling approach for extended multiscale FEM and PD was proposed for an efficient simulation of crack propagation in large scale solid structures [18]. Khodabakhshi et al. [19] proposed a non-local fracture criterion for a special type of FEA for eliminating the mesh sensitivity in results caused by a local fracture criterion. The proposed criterion can also be utilized within the traditional FEM.

A unification attempt was performed for reproducing kernel particle method and the state-based PD to utilize the advantages of both techniques to overcome issues associated with skin effects and non-uniform discretization in PD, see [20]. Smoothing property of Lanczos derivatives obtained by connecting state-based PD discretization and classical continuum mechanics was also discussed by the authors. Finally, the authors proposed improvements on the state-based PD addressing the issues associated with application of boundary conditions and nonuniform grids based on the findings from the study [20]. In the meshfree methods, the non-uniform particle arrangement for the demanded area was employed and mixed-mode SIFs of shear deformable plates [21-23] as well as buckling of cracked cylindrical and flat shells [24] were studied. In addition, same concept was recently applied to geometrical non-linear analysis of shells by a meshfree particle method [25]. Besides these works, the non-uniform particle discretization has been well established and applied in meshfree particle methods for several decades, see Refs. [26-28].

When employing different horizon sizes between the different scale particle distributions, "ghost" force arises along the transition region. In the present study, a novel variable horizon concept is presented. The gradual variation of the horizon size, called smoothing length, is newly introduced to suppress the ghost force along with the gradual variation of spatial resolution. The convenience of the smoothed variable horizon PD (SVH-PD) modeling is verified. First, PD formulation is investigated to treat the variable horizon. It is known that so-called Newton's third law in mechanics does not meet in the standard PD formulation when different horizon sizes are employed. Then, force states with different horizon sizes are examined and generalized. It is also shown that one of the formulation is identical with the DH-PD [11,12]. The multi-horizon PD [16] also addresses the ghost force effect, the present SVH-PD however seems to be more convenient from the $m$ convergence point of view. Moreover, the present work employs numerical length scale rather than the physical length scale [15], the present study therefore differs from a multiscale analysis.

As a basic study, we deal with simple problems without cracks to investigate influence of the variable horizon size. Then, two-dimensional (2D) stationary crack 
problems are examined for evaluating dynamic stress intensity factors (DSIFs) to quantify error in the PD modeling.

The structure of the paper is organized as follow. In section 2 , we briefly give the basics of a standard PD formulation. An arbitrary spatial resolution technique in the PD framework is described in section 3. In this section, the variable horizon concept is also explained considering the inconsistency of the interaction forces. The novelty of the present work, the smoothing length concept, is introduced in section 4. The numerical examples to discuss the accuracy and efficiency of the proposed approach are dealt with in section 5 . The concluding remarks are given in the last section.

\section{Peridynamic theory}

The basic formulation of PD theory can be derived from the equation of motion [29]. The equation of motion at position vector $\boldsymbol{x}$ in a finite body can be written in ordinary state-based PD (OSB-PD) framework as:

$$
\rho \ddot{\boldsymbol{u}}(\boldsymbol{x}, t)=\int_{\mathcal{H}_{x}}\left[\underline{\boldsymbol{T}}(\boldsymbol{x}, t)\left\langle\boldsymbol{x}^{\prime}-\boldsymbol{x}\right\rangle-\underline{\boldsymbol{T}}\left(\boldsymbol{x}^{\prime}, t\right)\left\langle\boldsymbol{x}-\boldsymbol{x}^{\prime}\right\rangle\right] \mathrm{d} V_{\boldsymbol{x}^{\prime}}+\boldsymbol{b}(\boldsymbol{x}, t),
$$

where $\rho$ is the material density, $\ddot{\boldsymbol{u}}(\boldsymbol{x}, t)$ is the acceleration vector, and $\boldsymbol{b}(\boldsymbol{x}, t)$ is the body force vector per unit volume. In the PD modeling, a finite body is discretized by particles. The particles located at $\boldsymbol{x}^{\prime}$ have a volume $\mathrm{d} V_{\boldsymbol{x}^{\prime}} \cdot \underline{\boldsymbol{T}}(\boldsymbol{x}, t)\left\langle\boldsymbol{x}^{\prime}-\boldsymbol{x}\right\rangle(=\underline{\boldsymbol{T}})$ and $\underline{\boldsymbol{T}}\left(\boldsymbol{x}^{\prime}, t\right)\left\langle\boldsymbol{x}-\boldsymbol{x}^{\prime}\right\rangle\left(=\underline{\boldsymbol{T}}^{\prime}\right)$ are the force states of particles located at $\boldsymbol{x}$ and $\boldsymbol{x}^{\prime}$, respectively, which are interacting with each other. A particle interacts with other particles within an influence radius $\delta . \mathcal{H}_{x}$ represents the neighborhood of the particle located at $\boldsymbol{x}$ as shown in Fig. 1.

(a)

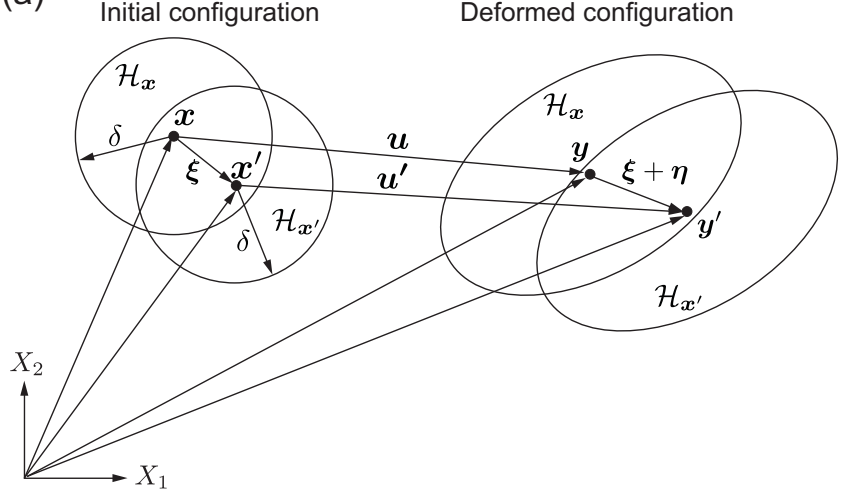

(b)

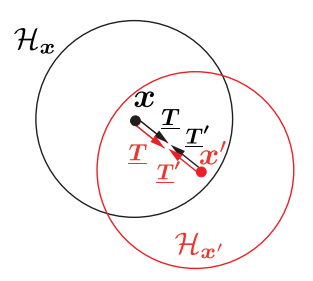

Fig. 1 A standard PD concept: (a) kinematics, (b) force states.

In the OSB-PD formulation, the force state vector $\underline{T}$ is expressed in terms of a scalar force density, $\underline{t}$ and a unit direction vector, $\boldsymbol{m}$ as [30]:

$$
\underline{T}=\underline{t} m,
$$


where $\boldsymbol{m}$ is defined in the deformed configuration as $\boldsymbol{m}=(\boldsymbol{\xi}+\boldsymbol{\eta}) /|\boldsymbol{\xi}+\boldsymbol{\eta}| \cdot \boldsymbol{\xi}(=$ $\left.\boldsymbol{x}-\boldsymbol{x}^{\prime}\right)$ and $\boldsymbol{\eta}\left(=\boldsymbol{u}-\boldsymbol{u}^{\prime}\right)$ are the relative position and displacement vectors, respectively. The scalar force density, $\underline{t}$ is given for the plane stress condition as $[30]$ :

$$
\underline{t}=\frac{2(2 \nu-1)}{\nu-1}\left(\kappa^{\prime} \theta-\frac{\alpha}{3}\left(\underline{\omega e}^{\mathrm{d}}\right) \bullet|\underline{\boldsymbol{\xi}}|\right) \frac{\underline{\omega}|\underline{\boldsymbol{\xi}}|}{(\underline{\omega} \mid \underline{\boldsymbol{\xi}}) \bullet|\underline{\boldsymbol{\xi}}|}+\alpha \underline{\omega e}^{\mathrm{d}} .
$$

In the given expression, "•" represents a dot product of two states [29]. The volume dilatation, $\theta$ and other PD parameters, $\alpha$ and $\kappa^{\prime}$, can be expressed as:

$$
\begin{gathered}
\theta=\frac{2(2 \nu-1)}{\nu-1} \frac{(\underline{\omega}|\underline{\boldsymbol{\xi}}|) \bullet \underline{e}}{(\underline{\omega}|\underline{\boldsymbol{\xi}}|) \bullet|\underline{\boldsymbol{\xi}}|} \\
\alpha=\frac{8 G}{(\underline{\omega}|\underline{\boldsymbol{\xi}}|) \bullet|\underline{\boldsymbol{\xi}}|}, \\
\kappa^{\prime}=K+\frac{G(\nu+1)^{2}}{9(2 \nu-1)^{2}} .
\end{gathered}
$$

The magnitude of a scalar force density, $\underline{t}$ for the plane strain condition is

$$
\underline{t}=2\left(\kappa^{\prime} \theta-\frac{\alpha}{3} \underline{\omega e}^{\mathrm{d}} \bullet|\underline{\boldsymbol{\xi}}|\right) \frac{\underline{\omega}|\underline{\boldsymbol{\xi}}|}{(\underline{\omega}|\underline{\boldsymbol{\xi}}|) \bullet|\underline{\boldsymbol{\xi}}|}+\alpha \underline{\omega e^{\mathrm{d}}}
$$

where the parameters are

$$
\begin{gathered}
\theta=2 \frac{(\underline{\omega}|\underline{\xi}|) \bullet \underline{e}}{(\underline{\omega}|\underline{\boldsymbol{\xi}}|) \bullet|\underline{\boldsymbol{\xi}}|}, \\
\alpha=\frac{8 G}{(\underline{\omega}|\underline{\boldsymbol{\xi}}|) \bullet|\underline{\boldsymbol{\xi}}|}, \\
\kappa^{\prime}=K+\frac{G}{9} .
\end{gathered}
$$

In the above equations, $\nu, G$ and $K$ are Poisson's ratio, the elastic shear and bulk modulus, respectively. $\underline{\omega}$ is the influence function, which is a non-dimensional measure of the distance between the particles. The influence function is considered to be zero outside the horizon. $\underline{e}^{\mathrm{d}}$ is deviatoric part of the extension state, $\underline{e}$.

\section{Arbitrary spatial resolution technique}

Computational efficiency is crucial in any numerical methods for efficient use of computational resources. The computational cost in FEM mostly depends on the mesh size and the number of elements (degrees of freedom). In FEM, a detailed fine mesh is generally required for a stress concentration region where the displacement/stress gradient changes remarkably, and the computational cost is reduced by generating coarse mesh in the regions where the displacement gradient is smooth. However, it is difficult for a standard PD to deal with models utilizing different spatial resolutions unlike FEM. Nikravesh and Gerstle [31] utilized different lattice orientations for the purpose of showing variable lattice resolution in improved state-based lattice PD. In this section, a standard PD that allows different spatial resolutions is formulated. 
$3.1 m$-convergence and $\delta$-convergence

In the mesh-based methods, e.g., FEM, it is widely known that the element size and the order of the approximate function (linear or higher-order elements) affect the numerical accuracy. On the other hand, the horizon size, discretization size, number of neighboring particles, and the weight function have significant influence on the numerical accuracy of the PD simulation. Bobaru et al. [32] studied adaptive and uniform refinement for the one-dimensional PD. It was reported that the adaptive refinement produces significantly lower error than the uniform refinement for the same number of nodes [32]. They also stated that accuracy in the PD modeling has been improved by reducing the horizon size $\delta$ on the condition that enough number of particles inside the horizon exists. Cheng et al. [33] also carried out $m$-convergence and $\delta$-convergence studies for dynamic fracture analysis of shale material using PD.

(a)

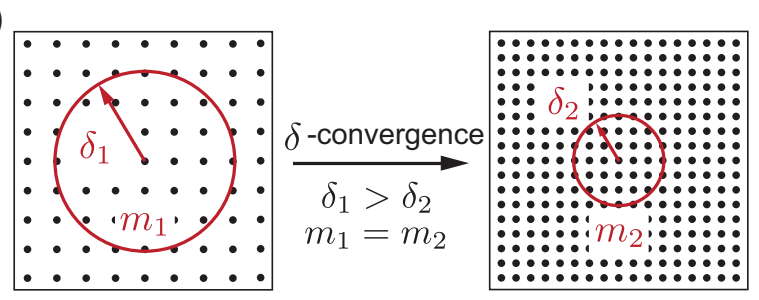

(b)

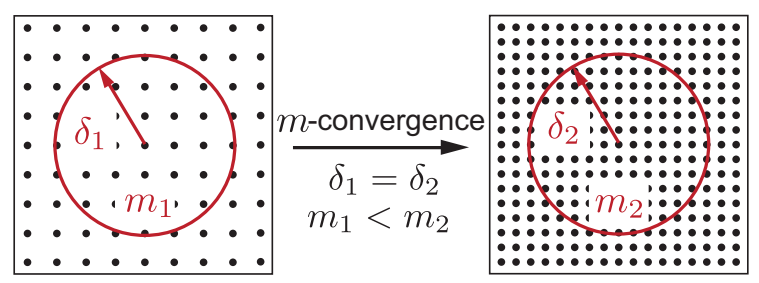

Fig. 2 Schematics of $\delta$ - $m$-convergence: (a) $\delta$-convergence, (b) $m$-convergence.

In the literature, defining the convergence for horizon size as $\delta$-convergence, and the convergence for the number of neighboring particles as $m$-convergence; it is necessary to pay attention to $\delta$-convergence while maintaining sufficient $m$ to achieve convergence of the solution. The $\delta$ - $m$-convergence concept is schematically shown in Fig. 2. Fig. 2(a) shows $\delta$-convergence representing change of horizon size while maintaining the same number of particles inside the horizon. On the other hand, Fig. 2(b) illustrates the $m$-convergence performed by changing the number of particles inside the horizon but keeping the horizon size constant.

\subsection{Inconsistency of interaction forces}

As inferred from $m$-convergence and $\delta$-convergence, it is considered that the variable horizon size as well as the spatial resolution of the particles are important to 
improve the analysis efficiency. However, the standard PD formulation does not locally satisfy so-called Newton's third law when the different horizon sizes are utilized. Fig. 3(a) shows the constant horizon size in the standard PD. In this case, both of the particles are the neighbor of each other. Here, the particle group $\mathcal{N}_{(i)}^{\mathcal{P}}$ in horizon $\delta_{i}$ of particle $(i)$ can be represented as follows.

$$
\mathcal{N}_{(i)}^{\mathcal{P}}=\mathcal{H}_{(i)}=\left\{|\boldsymbol{\xi}|<\delta_{(i)}\right\}
$$

In this case, the PD equation of motion for a particle $(i)$ can be discretized as:

$$
\rho \ddot{\boldsymbol{u}}_{(i)}=\sum_{j}^{\mathcal{N}_{(i)}^{\mathcal{P}}}\left[\underline{\boldsymbol{T}}_{(i)(j)}-\underline{\boldsymbol{T}}_{(j)(i)}\right] V_{(j)}+\boldsymbol{b}_{(i)} .
$$
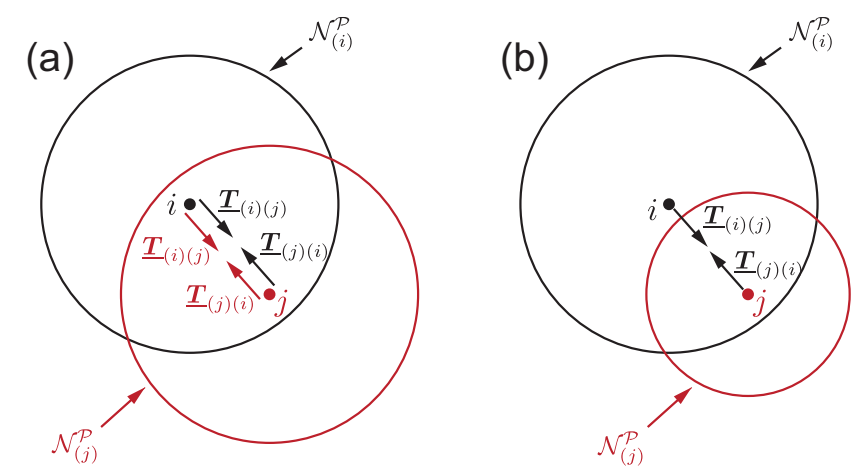

Fig. 3 Force states: (a) $x_{(i)} \in \mathcal{N}_{(j)}^{\mathcal{P}}$ and $x_{(j)} \in \mathcal{N}_{(i)}^{\mathcal{P}}$, (b) $x_{(i)} \notin \mathcal{N}_{(j)}^{\mathcal{P}}$ and $x_{(j)} \in \mathcal{N}_{(i)}^{\mathcal{P}}$.

Fig. 3(a) shows that the particles $(i)$ and $(j)$ have the same horizon size $\delta_{(i)}=$ $\delta_{(j)}$, which corresponds to the standard PD. The particles at $\boldsymbol{x}_{(i)}$ and $\boldsymbol{x}_{(j)}$ fall into the neighborhood domain of each other, $\boldsymbol{x}_{(i)} \in \mathcal{N}_{(j)}^{\mathcal{P}}$ and $\boldsymbol{x}_{(j)} \in \mathcal{N}_{(i)}^{\mathcal{P}}$. The corresponding force states are then expressed as:

$$
\begin{cases}\underline{\boldsymbol{f}}_{(i)(j)}=\underline{\boldsymbol{T}}_{(i)(j)}-\underline{\boldsymbol{T}}_{(j)(i)} & \text { for } \quad i \text {-th particle } \\ \underline{\boldsymbol{f}}_{(j)(i)}=\underline{\boldsymbol{T}}_{(j)(i)}-\underline{\boldsymbol{T}}_{(i)(j)} & \text { for } \quad j \text {-th particle. }\end{cases}
$$

Not surprisingly, Newton's third law is satisfied as given below.

$$
\underline{\boldsymbol{f}}_{(i)(j)}=-\underline{\boldsymbol{f}}_{(j)(i)}
$$

Fig. 3(b) shows the case that the horizon sizes of particles $(i)$ and $(j)$ are not the same, $\delta_{(i)} \neq \delta_{(j)}$. While the particle $(j)$ falls into the neighborhood of the particle $(i)$, the particle $(i)$ is not covered by the horizon of particle $(j), \boldsymbol{x}_{(i)} \notin \mathcal{N}_{(j)}^{\mathcal{P}}$ and $\boldsymbol{x}_{(j)} \in \mathcal{N}_{(i)}^{\mathcal{P}}$. The corresponding force states become as:

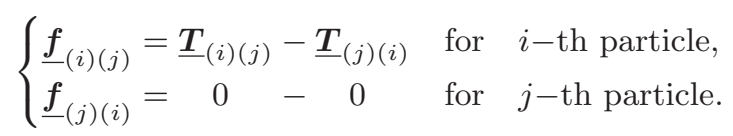


The relations above do not satisfy Newton's third law.

$$
\underline{\boldsymbol{f}}_{(i)(j)} \neq-\underline{\boldsymbol{f}}_{(j)(i)}
$$

3.3 Variable horizon concept

\subsubsection{Formulation}

As discussed in the previous section, when the horizon size is spatially varied, the third law of Newton is not ensured. This concept without any special treatments is therefore not applicable for the problems, it is necessary to satisfy the Newton's third law in mechanics. In order to clarify this requirement for the particles with different horizon sizes, the cases shown in Fig. 4 are considered. Fig. 4(a)(d) schematically illustrate the different force states (Cases A-D) with variable horizons, respectively.
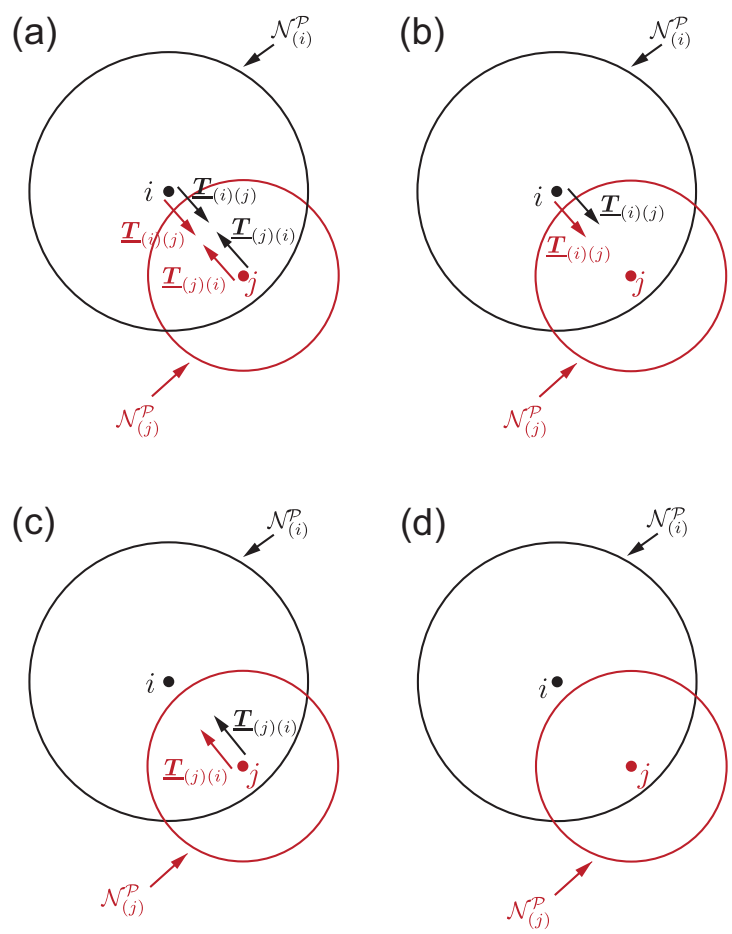

Fig. 4 Force state cases: (a) Case A, (b) Case B, (c) Case C, (d) Case D.

Case A:

$$
\left\{\begin{array}{lll}
\underline{\boldsymbol{f}}_{(i)(j)}=\underline{\boldsymbol{T}}_{(i)(j)} & -\underline{\boldsymbol{T}}_{(j)(i)} & \text { for } i \text {-th particle } \\
\underline{\boldsymbol{f}}_{(j)(i)}=\underline{\boldsymbol{T}}_{(j)(i)} & -\underline{\boldsymbol{T}}_{(i)(j)} & \text { for } j \text {-th particle. }
\end{array}\right.
$$


Case B:

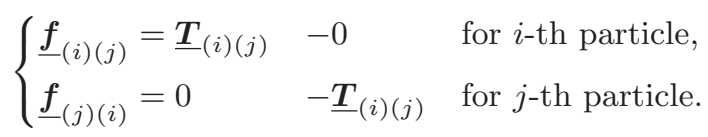

Case C:

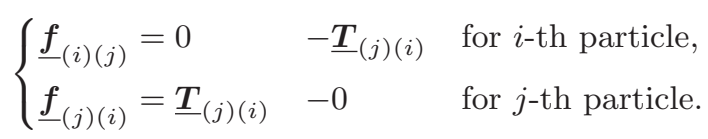

Case D:

$$
\left\{\begin{array}{lll}
\underline{\boldsymbol{f}}_{(i)(j)}=0 & -0 & \text { for } i \text {-th particle } \\
\underline{\boldsymbol{f}}_{(j)(i)}=0 & -0 & \text { for } j \text {-th particle. }
\end{array}\right.
$$

In all cases given in Fig. 4, Newton's third law is satisfied, and the theory holds for current PD discretization. In Fig. 4, the influence functions $\omega_{(i)(j)}$ and $\omega_{(j)(i)}$ are expressed as:

$$
\begin{array}{ll}
\omega_{(i)(j)} \neq 0 & \text { for } \boldsymbol{x}_{(j)} \in \mathcal{N}_{(i)}^{\mathcal{P}}, \\
\omega_{(j)(i)}=0 & \text { for } \boldsymbol{x}_{(i)} \notin \mathcal{N}_{(j)}^{\mathcal{P}} .
\end{array}
$$

According to this definition, the force states for Cases A-D are rewritten as follows.

Case A:

$$
\left\{\begin{array}{lll}
\underline{\boldsymbol{f}}_{(i)(j)}=\underline{\boldsymbol{T}}_{(i)(j)} & -0 & \text { for } i \text {-th particle } \\
\underline{\boldsymbol{f}}_{(j)(i)}=0 & -\underline{\boldsymbol{T}}_{(i)(j)} & \text { for } j \text {-th particle. }
\end{array}\right.
$$

Case B:

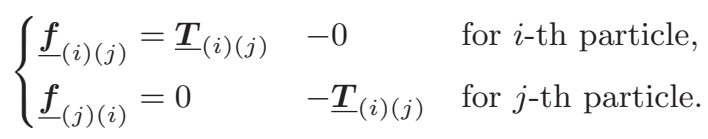

Case C:

$$
\left\{\begin{array}{lll}
\underline{\boldsymbol{f}}_{(i)(j)}=0 & -0 & \text { for } i \text {-th particle } \\
\underline{\boldsymbol{f}}_{(j)(i)}=0 & -0 & \text { for } j \text {-th particle. }
\end{array}\right.
$$

Case D:

$$
\left\{\begin{array}{lll}
\underline{\boldsymbol{f}}_{(i)(j)}=0 & -0 & \text { for } i \text {-th particle } \\
\underline{\boldsymbol{f}}_{(j)(i)}=0 & -0 & \text { for } j \text {-th particle. }
\end{array}\right.
$$

The above expressions state that Cases A and B are the same when the ordinary influence function is adopted. It is considered that the Case B is appropriate for representing the transmission of the interaction forces. This is also consistent with the formulation of DH-PD [11]. Assume the influence function for particles $(i)$ and $(j)$ is redefined as:

$$
\omega_{(i)(j)}=\omega_{(j)(i)}=\max \left(\omega_{(i)(j)}, \omega_{(j)(i)}\right) .
$$

Cases A, B and C can support different horizon sizes utilizing the expression in Eq. (27). As described above, in order to adopt different horizon sizes, it is necessary 
to employ a different formulation from the force state of the standard PD, and the equation of motion can be expressed in discretized form as:

$$
\rho \ddot{\boldsymbol{u}}_{(i)}=\sum_{j}^{\mathcal{N}_{(i)}^{\mathcal{V H}}} \underline{\boldsymbol{T}}_{(i)(j)} V_{(j)}+\sum_{j}^{\mathcal{N}_{(i)}^{\mathcal{\nu} \mathcal{H}^{\prime}}} \underline{\boldsymbol{T}}_{(j)(i)} V_{(j)}+\boldsymbol{b}_{(i)},
$$

where

$$
\begin{aligned}
\mathcal{N}_{(i)}^{\mathcal{V H}} & =\left\{\boldsymbol{x} \in \mathcal{B} \mid \underline{\omega}_{(i)(j)} \neq 0\right\}, \\
\mathcal{N}_{(i)}^{\mathcal{V} \mathcal{H}^{\prime}} & =\left\{\boldsymbol{x} \in \mathcal{B} \mid \underline{\omega}_{(j)(i)} \neq 0\right\} .
\end{aligned}
$$

Here, we define Eq. (28) as generalized VH-PD.

\subsection{Verification of the variable horizon concept}

In order to present validity of the Eq. (28), the stress induced wave propagation problem in a plate shown in Fig. 5 is considered. Two types of particle arrangements as depicted in Fig. 6 are compared using the standard PD and the generalized VH-PD, Eq. (28). The reference solution is Case A at which the horizon size, as shown in Fig. 6, is constant throughout the model. Target points are enumerated as \#1, \#2 and \#3 in Fig. 5. The material properties are taken as Young's modulus $E=210 \mathrm{GPa}$, Poisson's ratio $\nu=1 / 3$, the material density $\rho=7,850$ $\mathrm{kg} / \mathrm{m}^{3}$. The plane stress condition is utilized.

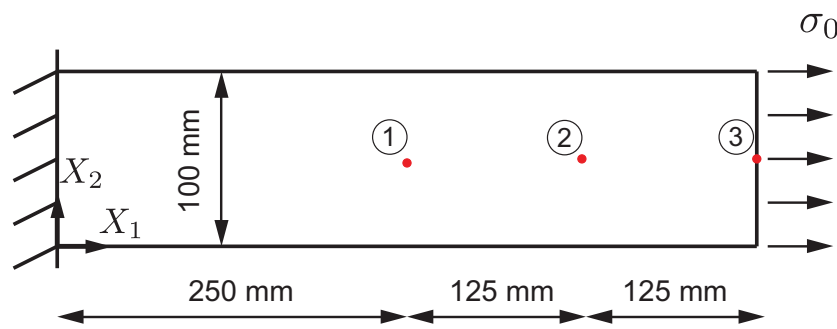

Fig. 5 A rectangular plate subjected to a tensile load.

A uniform tensile stress of magnitude $\sigma_{0}=0.667 \mathrm{MPa}$ is suddenly applied to the right edge of the specimen, see Fig. 5. The horizon sizes are defined as $\delta_{1}=3.5 d l_{1}, \delta_{2}=3.5 d l_{2}$ and the distance between the particles for coarse and fine regions are $d l_{1}=5.0, d l_{2}=2.5 \mathrm{~mm}$, respectively. The discretization is depicted in Fig. 6. In both horizon size cases, the number of particles inside the neighborhood is constant.

Models $\mathrm{A}$ and $\mathrm{B}$ are examined by employing the standard PD and the generalized VH-PD. The displacement magnitudes for points \#1, \#2 and \#3 are measured for total duration of $0.5 \mathrm{~ms}$. It must be noted that the standard PD equation of motion is given by Eq. (12), while the generalized VH-PD equation of motion is expressed by Eq. (28). Here, the comparison is carried out for these two approaches adopting different particle discretizations. 


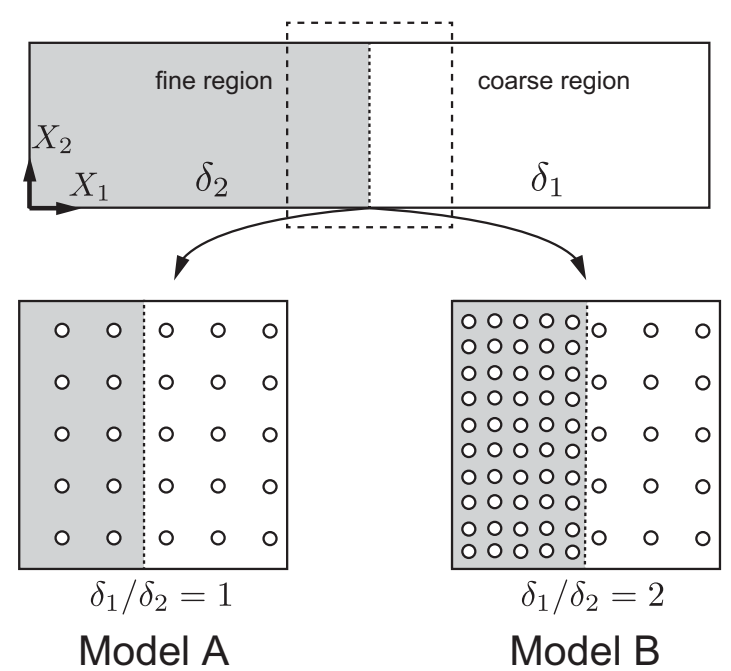

Fig. 6 Particle arrangements for Models A and B.

Fig. 7(a) shows the analysis results of Models A and B by the standard PD, Eq. (12). It can be seen that the results of Model B, in which the horizon is not constant, do not obviously match the results of Model A of the constant horizon over the entire model, which is an outcome of inconsistency in the interaction forces. In both points, the peaks for displacements are underestimated and the occurrence instance of peaks also do not match with the Model A. The deviation from the Model A is more pronounced for the points \#2 and \#3, where located on the coarse region. This could be explained by the spurious wave reflection from the interface of the different horizon sizes. On the other hand, Fig. 7(b) shows that the analysis results perfectly match for both Models A and B, indicating that an appropriate solution is obtained even when the horizon size is varied. Therefore, validity of the VH-PD concept, Eq. (28) is confirmed.

(a)

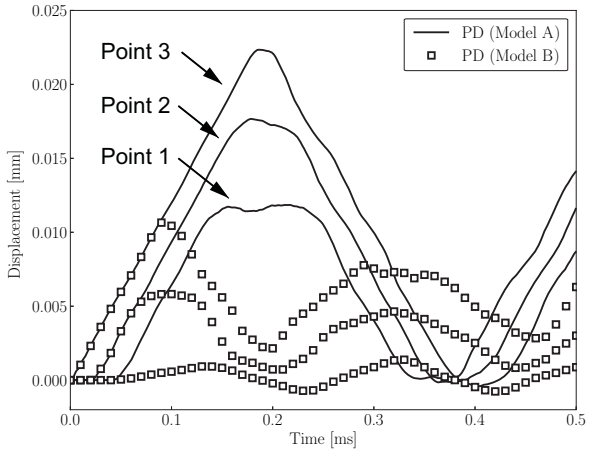

(b)

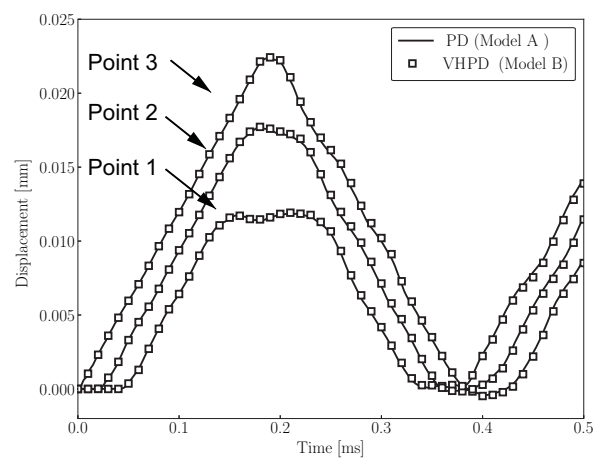

Fig. 7 Comparison between Models A and B by: (a) standard PD, (b) VH-PD. 


\subsection{Ghost forces}

In this section, generation of an undesired artificial force, i.e., ghost force arising due to the different horizon sizes of the mutual particles, is briefly described according to Ref. [8]. The ghost force that originally depends on the reference interaction force $\boldsymbol{T}_{1}$ for the equilibrium state, $\boldsymbol{L}=0$ is written as:

$$
\begin{aligned}
\boldsymbol{L}(\boldsymbol{x}) & =-\frac{1}{2} \int \nabla \nabla \delta(\boldsymbol{x})(\boldsymbol{m} \otimes \boldsymbol{m}) \underline{\boldsymbol{T}}_{1}\langle\boldsymbol{m}\rangle \mathrm{d} V_{\boldsymbol{m}}+\ldots \\
& =O(|\nabla \nabla \delta|) O\left(\left|\underline{\boldsymbol{T}}_{1}\right|\right) .
\end{aligned}
$$

The error term in Eq. (30) states that the ghost force is related to second derivative of the horizon size and the magnitude of the reference interaction force, which also proves that the ghost force would not exist if the horizon size is constant in the entire model.

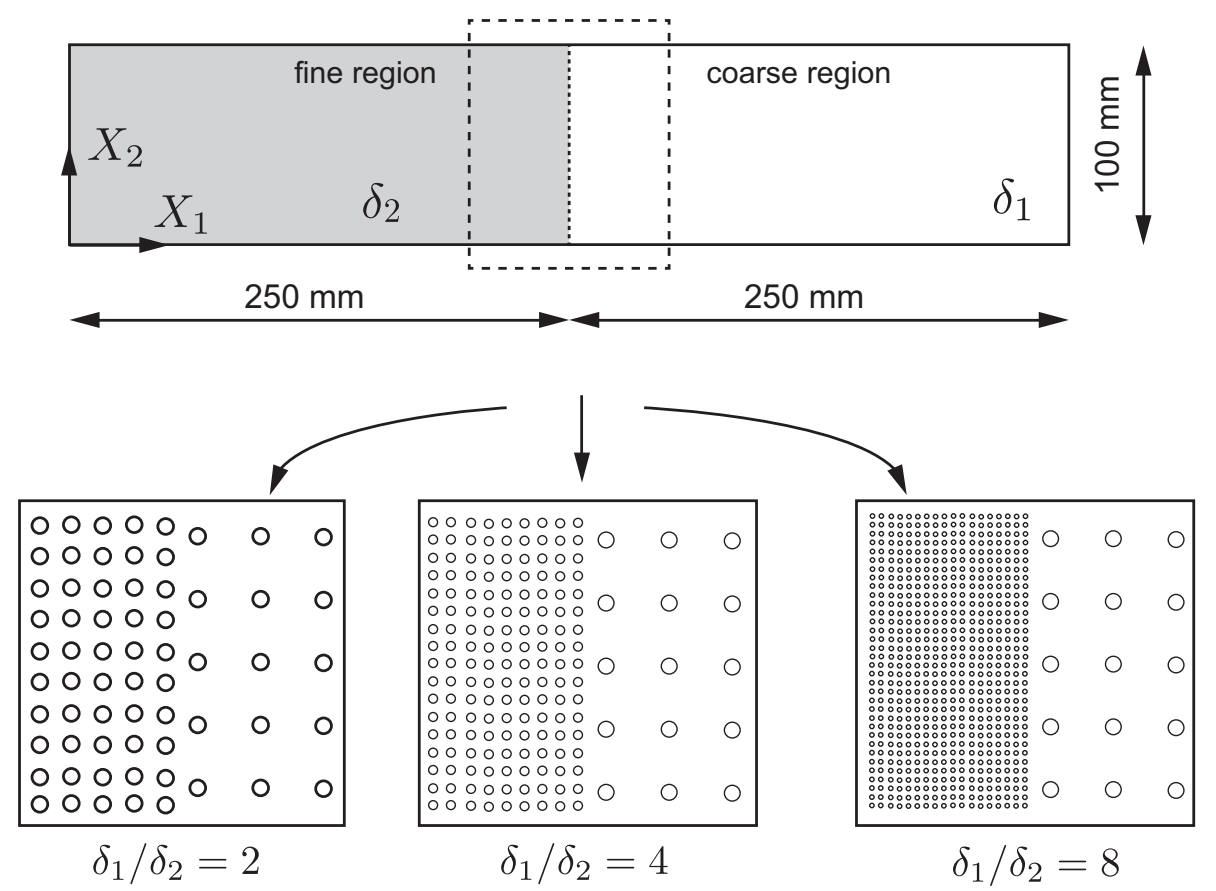

Fig. 8 A rectangular plate modeled by different horizons.

\subsection{Validation of the ghost force}

In order to verify influence of the ghost force on the internal force density, a displacement field is applied to a rectangular plate model shown in Fig. 8. Keeping the $m$ value constant, the distance between the particles as well as the horizon size are varied at the center of the plate (interface of the different spatial resolutions), 
see Fig. 8. The horizon size ratio $\delta_{1} / \delta_{2}$ is varied as 2.0, 4.0 and 8.0, respectively. Internal force density is considered as the indicator. In this example, inertia and external forces are omitted; this is because a forced linear displacement field is adopted, which is expressed as:

$$
u_{1}=\frac{\partial u_{1}}{\partial X_{1}} X_{1}
$$

Therefore, the analytical solution for the internal force density is $\boldsymbol{L}=0$.

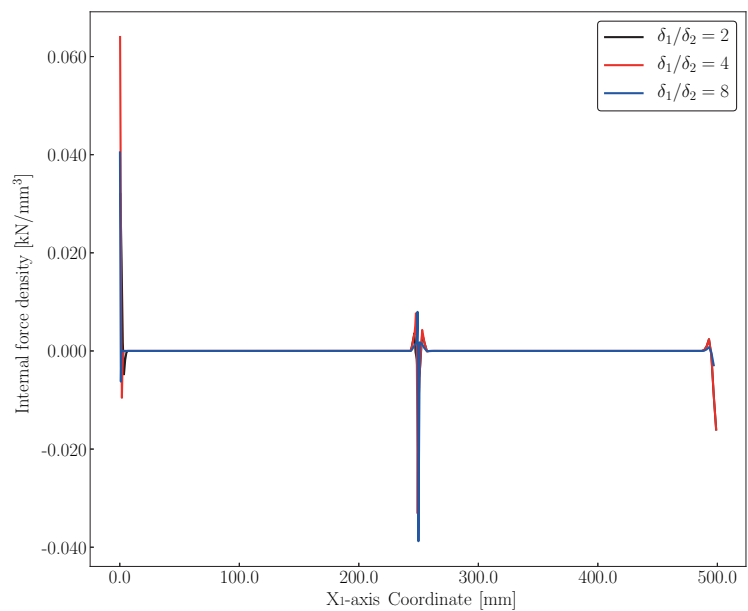

Fig. 9 Comparison of internal force densities for the different horizon size ratios, $\delta_{1} / \delta_{2}$.

Fig. 9 shows the variation of internal force densities along the $X_{1}$ axis for the different horizon size ratios. It is obvious that the values are non-zero at the ends and the center of the specimen. The non-zero internal force densities at the both ends of the specimen are associated with surface (skin) effects. The surface effects arise from the nature of the PD formulation, but not due to the variable horizon. On the other hand, the numerical oscillation at the center of the model, where horizon size is suddenly changed, is because of the ghost force generation. As the horizon ratio $\delta_{1} / \delta_{2}$ increases, the ghost force becomes large and Eq. (30) is reproduced faithfully.

\section{Introduction of a smoothing length concept for variable horizon}

A model to reduce ghost force is proposed in this section. From the numerical analysis results in the previous sections and Eq. (30), it can be seen that the ghost force depends on $\nabla \nabla \delta$ and $T_{1}$, see Eq. (30). It can be easily inferred that the ghost force can be reduced by smoothly changing the horizon size gradient in the region, where the spatial resolution is varied. In this section, "smoothing length $\left(L_{s}\right)$ concept" as shown in Fig. 10 is proposed. In this model, the horizon size is not suddenly varied. The smoothing length, $L_{s}$ denotes the length of the region where the horizon size is gradually varied, see Fig. 10. The smooth transition between 
the different horizon sizes reduces the error term $(|\nabla \nabla \delta|)$ of the internal force densities in Eq. (30).

(a)

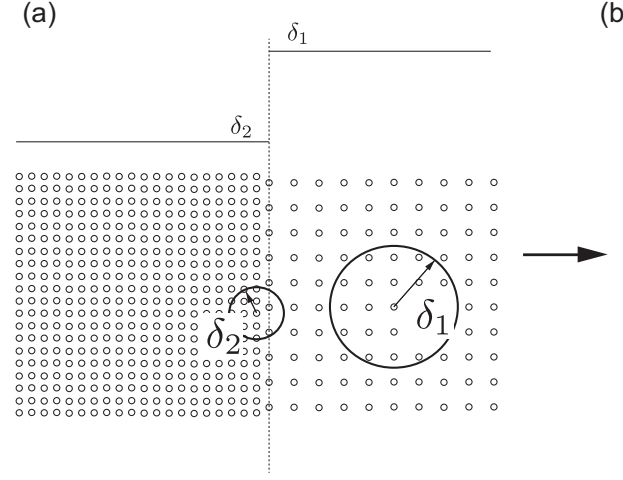

(b)

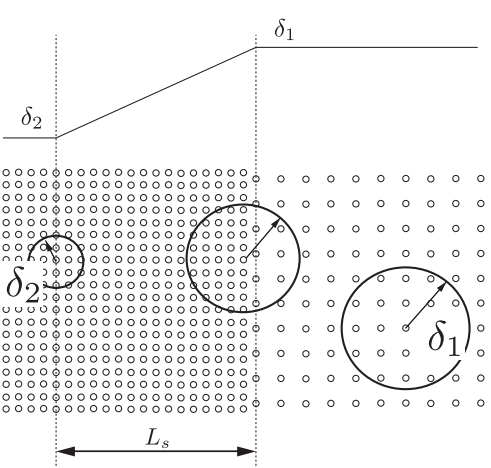

Fig. 10 Definition of the smoothing length: (a) sudden change of horizon sizes, (b) model with the smoothing length concept for gradual variation of horizon sizes.

To examine the effectiveness of the smoothing length concept, the previous numerical model shown in Fig. 8 is considered. Different smoothing lengths of $L_{s}=0, L_{s}=5 d l_{1}$ and $L_{s}=10 d l_{1}$ are adopted to observe the oscillation of internal force density. Note that $L_{s}=0$ is the same as the previous section, where the smoothing length is not introduced; the horizon size changes rapidly at the interface.

The influence of the smoothing length concept is illustrated in Fig. 11(a)-(c) for smoothing lengths $L_{s}=0, L_{s}=5 d l_{1}$ and $L_{s}=10 d l_{1}$, respectively. The given figure shows that the internal force density is reduced by increase of the smoothing length. It is clear that the magnitude of ghost force becomes smaller by the smoothing approach and the oscillations are redistributed over the smoothing region with a small magnitude, which mostly occurs at the left boundary of the smoothing region (smaller horizons). Here, the approach is called as smoothed variable horizon PD (SVH-PD).

\section{Numerical examples}

The SVH-PD is employed to calculate DSIFs of stationary cracks in this section. The DSIFs evaluation techniques in PD modeling were previously proposed by the authors $[3,4]$. The evaluation techniques are briefly revisited here. At first, the $J$ integral method for evaluating DSIFs is described. Then, the path independence of DSIFs in SVH-PD framework is studied. Finally, the DSIFs obtained by the present approach are presented comparing with the analytical reference results. The reduction of the computational cost by SVH-PD is clearly indicated. 

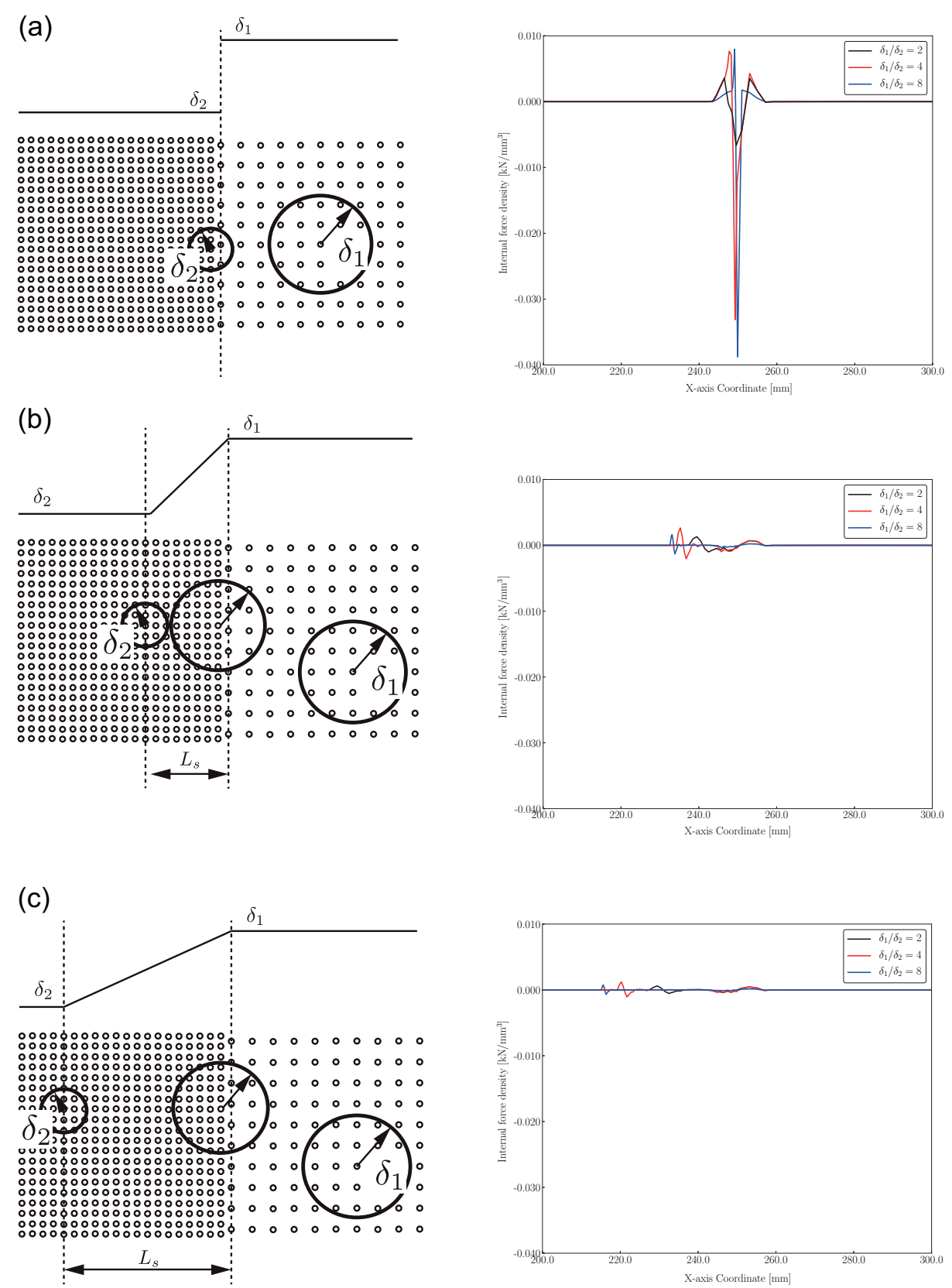

Fig. 11 Comparison of internal force densities for: (a) $L_{s}=0$, (b) $L_{s}=5 d l_{1}$, (c) $L_{s}=10 d l_{1}$.
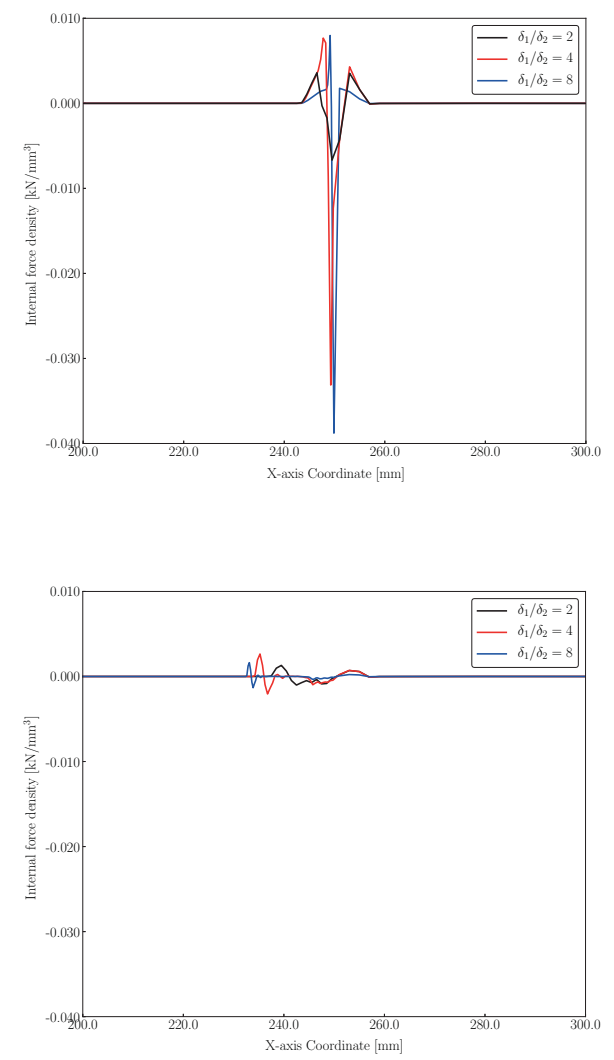
5.1 J-integral method for evaluating DSIFs

Path independent energy release rate is calculated near the crack tip using the $J$ integral proposed by Rice [34]. The $J$-integral approach is schematically illustrated in Fig. 12.

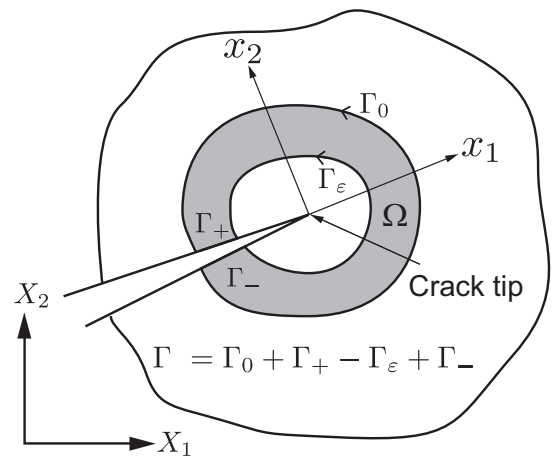

Fig. 12 A schematic illustration of $J$-integral domain.

The original form of the $J$-integral is a path integral. Here, we take the dynamic effects into account. Employing the Gauss divergence theorem, the $J$-integral can be expressed as domain $\Omega$ integration [35-37], which is written below.

$$
J=\int_{\Omega}\left[\left(\sigma_{i j} \frac{\partial u_{i}}{\partial x_{1}}-W \delta_{1 j}\right) \frac{\partial q}{\partial x_{j}}+\rho \frac{\partial^{2} u_{i}}{\partial t^{2}} \frac{\partial u_{i}}{\partial x_{1}} q\right] \mathrm{d} \Omega
$$

In the given equation, $x_{1}$ and $x_{2}$ are local coordinate axes respectively parallel and normal to the crack segment, whose origin is located at the crack tip. $\sigma_{i j}, u_{i}$ and $\rho$ are the stress, displacement, and material density components. $W$ and $\delta_{1 j}$ stand for the strain energy density and Kronecker delta, respectively. $q$ is an arbitrary continuous function that becomes zero at the outer boundary, $\Gamma_{0}$ and unity at the inner boundary, $\Gamma_{\varepsilon}$ of the domain $\Omega$. To ensure the path independence, circular or rectangular integration domains can be utilized.

The opening mode DSIF, $K_{\mathrm{I}}^{\mathrm{d}}$ is evaluated from the energy release rate $J$ as follow,

$$
K_{\mathrm{I}}^{\mathrm{d}}=\sqrt{J E^{\prime}},
$$

where $E^{\prime}$ is a material property as $E^{\prime}=E$ and $E^{\prime}=E /\left(1-\nu^{2}\right)$ for plane stress and plane strain conditions, respectively.

In computing the DSIFs by the $J$-integral, it is required to evaluate the displacement gradients. The current form of the PD cannot calculate the displacement gradients directly, the Moving Least Squares (MLS) method [38] is therefore adopted to approximate the displacement gradients of the particles.

As for the numerical integration of the energy release rate $J$, the displacement components are calculated in PD framework and introduced into the MLS approximation. The discretized form of the $J$-integral is then expressed as:

$$
J=\sum_{k}^{\Omega}\left[\left\{\left(\sigma_{i j}\right)_{k}\left(\frac{\partial u_{i}}{\partial x_{1}}\right)_{k}-W_{k} \delta_{1 j}\right\}\left(\frac{\partial q}{\partial x_{j}}\right)_{k}+\rho_{k}\left(\frac{\partial^{2} u_{i}}{\partial t^{2}}\right)_{k}\left(\frac{\partial u_{i}}{\partial x_{1}}\right)_{k} q_{k}\right] V_{k} .
$$


Here, the subscript $k$ stands for the $k$-th particle. $\left(\partial u_{i} / \partial x_{1}\right)_{k}$ is the displacement gradient obtained by MLS, which is expressed as:

$$
\left(\frac{\partial u_{i}}{\partial x_{1}}\right)_{k}=\sum_{l}^{N}\left(\frac{\partial \psi}{\partial x_{1}}\right)_{l}\left(u_{i}\right)_{l}
$$

where $\psi$ represents the shape function in the MLS approximation. $N$ is the total number of particles within the influence radius of the $k$-th particle. In the present study, the influence radius of MLS is utilized as same as the horizon size. Further details regarding the MLS approximation and its discretization can be found in Refs. [3,4]. The stress components in Eq. (34) are however computed within PD framework assuming the force flux at a particle is equivalent to state of stress, which is expressed as:

$$
\sigma=\int_{\mathcal{H}} \underline{\boldsymbol{T}} \otimes \underline{\boldsymbol{\xi}} \mathrm{d} V
$$

(a)

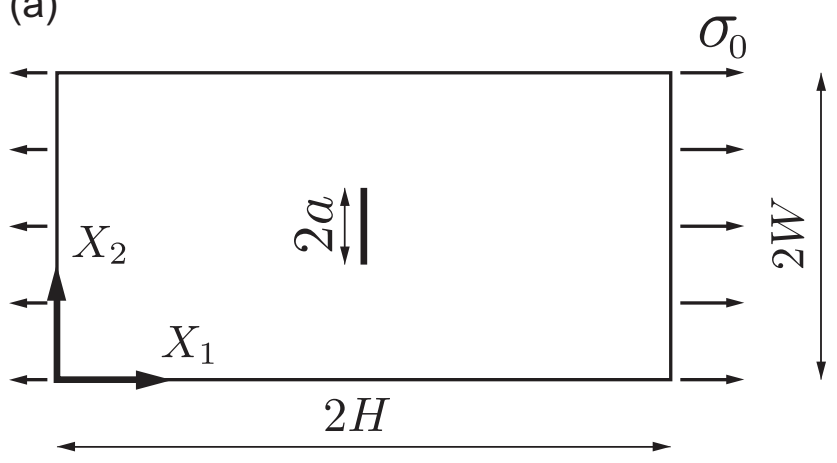

(c) $20 d l_{1}$

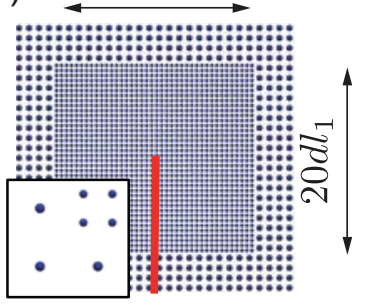

(d)

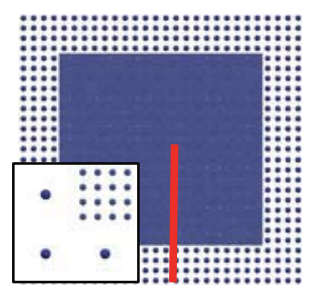

(b)

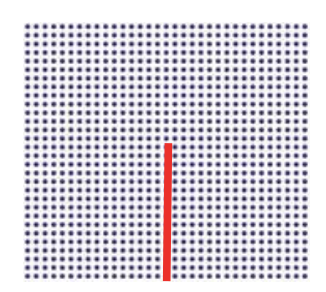

(e)

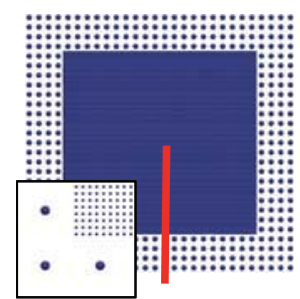

Fig. 13 Numerical model and particle arrangements around the crack tip: (a) a rectangular plate with a center crack, (b) particle arrangement for Level 0, (c) particle arrangement for Level 1, (d) particle arrangement for Level 2, (e) particle arrangement for Level 3.

5.2 Verification of the path independence

The DSIFs for a rectangular plate with a central crack shown in Fig. 13(a) are calculated to verify the path independence in the SVH-PD framework. The model 
dimensions are $2 H=40 \mathrm{~mm}, 2 W=20 \mathrm{~mm}, a=2.4 \mathrm{~mm}$, and the load $\sigma_{0}=1.0$ $\mathrm{GPa}$ is applied to both left and right edges of the rectangular plate. The material properties are taken as Young's modulus $E=199.992 \mathrm{GPa}$, material density $\rho=5,000 \mathrm{~kg} / \mathrm{m}^{3}$, and the Poisson's ratio $\nu=0.3$. The distance between the particles is set as $d l_{1}=0.1 \mathrm{~mm}$ in the coarse region and the particle distance in the fine region is varied depending on the horizon size ratio. The horizon size in both coarse and fine regions is taken as $\delta=4.0 \mathrm{dl}$.

As shown in Fig. 13, the crack tip region of dimension $20 d l_{1} \times 20 d l_{1}$ is discretized in high resolution. The difference between the resolutions is defined by levels. Level 0 represents the constant spatial resolution as well as the constant horizon size in the whole model, and therefore the smoothing length becomes zero for this model. The Levels 1, 2 and 3 stand for the horizon size ratio $\delta_{1} / \delta_{2}$ of 2.0, 4.0 and 8.0, respectively. $\delta_{2}$ denotes the horizon size in the crack tip (fine) region. The particle arrangements for these cases are respectively given in Fig. 13(c)-(e).

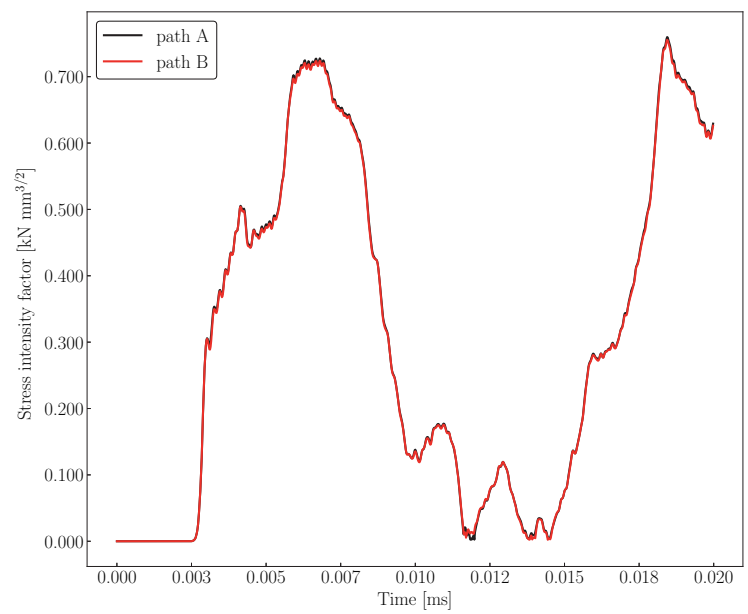

Fig. 14 DSIFs for paths A and B utilizing the constant horizon (Level 0).

Fig. 14 shows the DSIFs for constant horizon in the entire model as illustrated in Fig. 13(b). The path independence for the constant horizon case can thus be confirmed considering Fig. 14. Then, this case is utilized as the reference solution for the further verification of path independence in SVH-PD employing different spatial resolutions. Paths A and B have $J$-integration regions of $r_{1}^{q}=1.0 \mathrm{~mm}$, $r_{2}^{q}=2.0 \mathrm{~mm}$ and $r_{1}^{q}=0.5 \mathrm{~mm}, r_{2}^{q}=1.0 \mathrm{~mm}$, respectively. $r_{1}^{q}$ and $r_{2}^{q}$ stand for the distances from the crack tip to the inner and outer boundary of the domain, respectively. The following global error expression is considered as indicator to examine the path independence.

$$
\epsilon_{\mathrm{g}}=\frac{1}{|z|_{\max }} \sqrt{\frac{1}{N} \sum_{i}^{N}\left(z_{i}^{\mathrm{ref}}-z_{i}^{\mathrm{num}}\right)^{2}}
$$




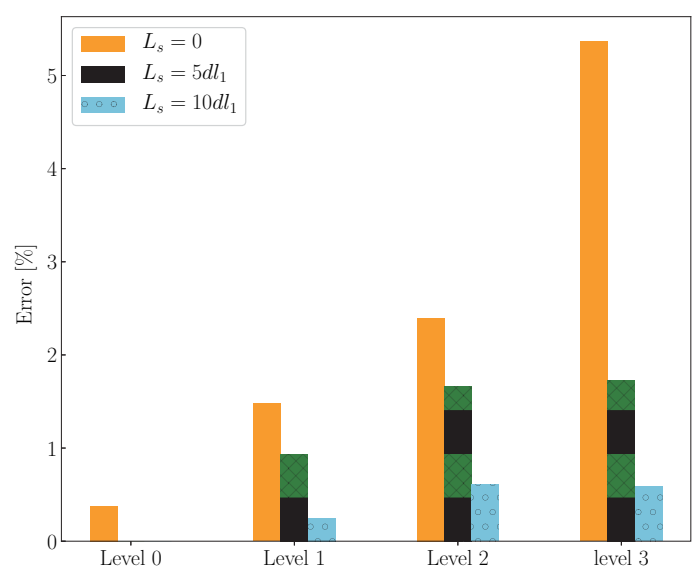

Fig. 15 Comparison of global error for the various smoothing lengths and the particle arrangements.

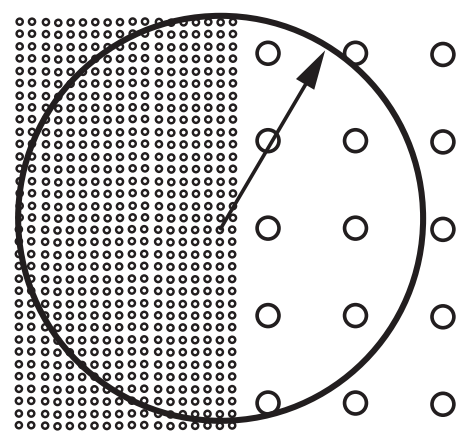

Fig. 16 The neighborhood of Level 3.

The global error for Level 0 is $0.60 \%$, and this value is used for the comparison reason. Fig. 15 shows the global error utilizing the smoothing lengths $L_{s}=0,5 d l_{1}$ and $10 d l_{1}$ for Levels $1-3$. In the $L_{s}=0$ case, it can be confirmed that the global error increases as Level $\left(\delta_{1} / \delta_{2}\right)$ increases. For each level, it is seen that the global error becomes smaller as the smoothing length $L_{s}$ increases, which obviously shows influence of the smoothing length on reducing the error in DSIFs. It is also clear in Fig. 15 that sufficient accuracy can be obtained with smoothing length concept even at Level 3, where the horizon size ratio is large. However, as shown in Fig. 16, the number of neighboring particles inside the horizon becomes very large, which is inefficient from the viewpoint of $m$-convergence. Therefore, we propose a method to take into account the $m$-convergence and enforce gradual variation of spatial resolution near the crack tip as shown in Fig. 17, called as step by step modeling. Fig. 18 indicates the results when the spatial resolution is gradually varied. The step by step modeling reveals the lowest error for the same smoothing length compared to Levels 1-3. 


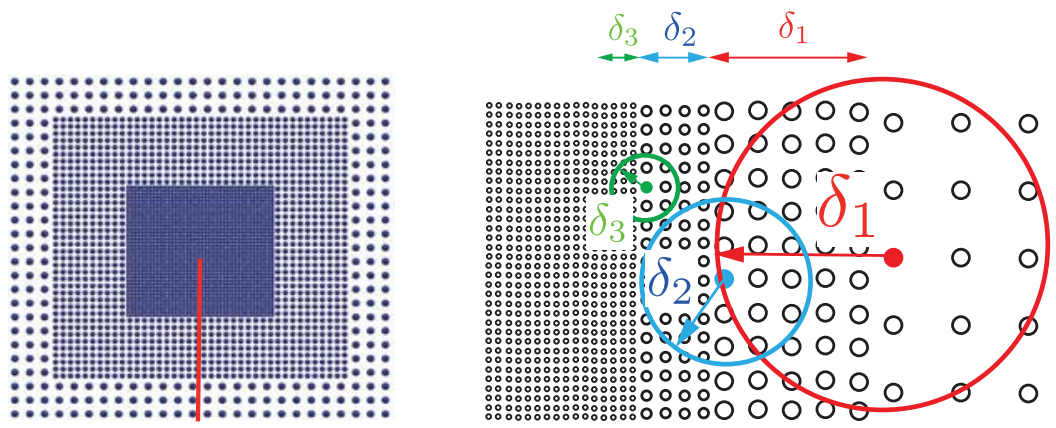

Fig. 17 A gradual variation of spatial resolution and horizon size.

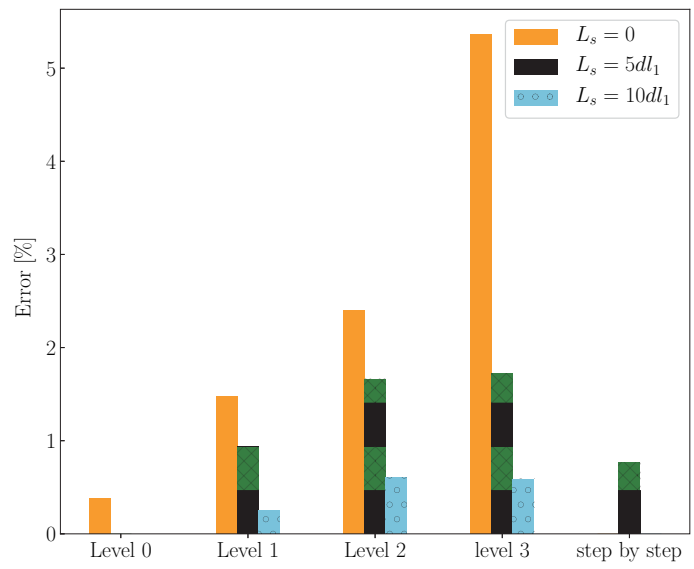

Fig. 18 Comparison of global error for the various smoothing lengths and the particle arrangements with step by step modeling.

\subsection{Evaluation of DSIFs for a stationary crack}

A semi-infinite plate with a through crack shown in Fig. 19(a) is considered. The model dimensions are $W=100 \mathrm{~mm}, 2 H=40 \mathrm{~mm}$, and $a=50 \mathrm{~mm}$. The material properties are utilized as Young's modulus $E=210 \mathrm{GPa}$, Poisson's ratio $\nu=0.3$, and the material density $\rho=7,850 \mathrm{~kg} / \mathrm{m}^{3}$. The plane strain condition is adopted. As a reference value, the analytical solution of a semi-infinite plate from Ref. [39] is considered, see Eq. (38).

$$
K_{\mathrm{I}}^{\mathrm{d}}= \begin{cases}0 & (t \leq \bar{t}), \\ 2 \sigma_{0} \frac{\sqrt{c_{d} t(1-2 \nu) / \pi}}{1-\nu} & (t>\bar{t}) .\end{cases}
$$

The total simulation time is $t<3 \bar{t}$ at which the semi-infinite condition is satisfied. $\bar{t}$ is the dimensionless time, which is expressed as:

$$
\bar{t}=c_{d} \times t / H,
$$

where $c_{d}$ is the longitudinal (dilatational) wave speed. $\bar{t}$ represents reaching time of a stress wave to the crack tip. 


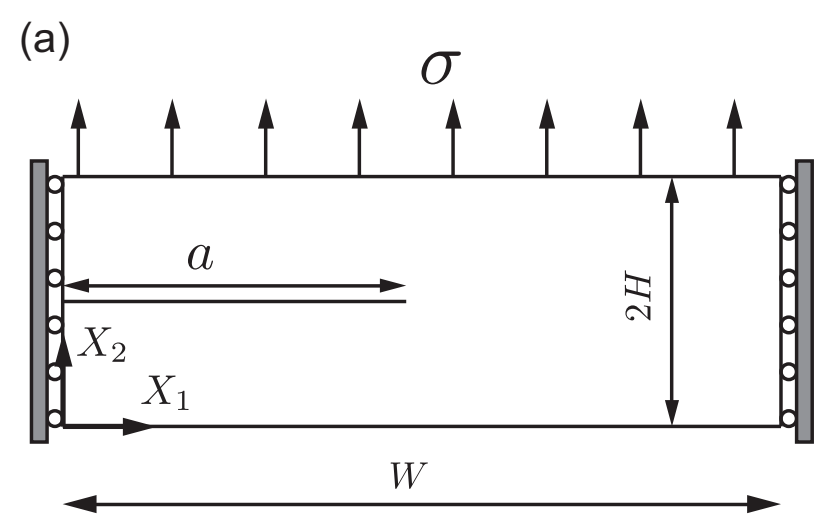

(b)

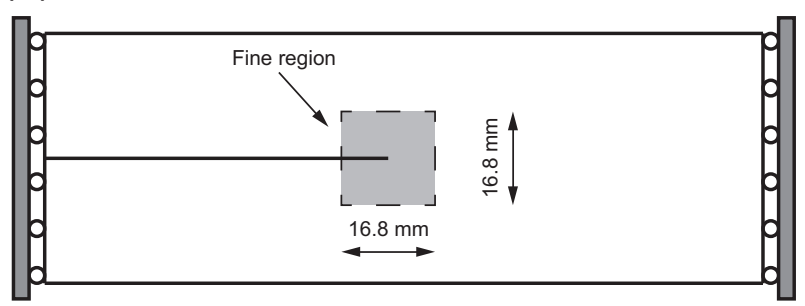

Fig. 19 Modeling for DSIFs evaluation: (a) semi-infinite rectangular plate model, (b) crack tip particle refinement.

For the SVH-PD implementation, the spatial resolution near the crack is further refined, see Fig. 19(b). The convergence of global error is examined with the standard PD and the SVH-PD. The distance between particles is varied as $d l=0.4,0.2,0.1,0.05 \mathrm{~mm}$, and the SVH-PD concept is applied from Level 0 to Level 3 as described in the preceding sections.

The results of the standard PD and the SVH-PD are shown in Figs. 20 and 21 , respectively. It can be confirmed that both results converge to the analytical solution. For further clarification purpose, close-up views are provided in Figs. 20(b)-(c) and 21(b)-(c). The close-up view for the standard PD indicates that $d l=0.05$ reveals the closest results to the analytical solution. As for the SVHPD approach, the closest result to the analytical solution is achieved for Level 3. The number of particles in the standard PD is 25,000, 100,000, 400,000, 1,600,000 for the particle distance values $d l=0.4,0.2,0.1,0.05 \mathrm{~mm}$, respectively. On the other hand, in the case of SVH-PD, the number of particles is $25,000,30,292$, 46,060, 99,124 for Levels 0-3, respectively. The given numbers clearly show that the efficiency is considerably improved. This phenomenon can also be observed in the global error plot of dimensionless DSIFs versus the number of particles for the standard PD and the SVH-PD, which is given in Fig. 22. The smallest error is immediately achieved in the SVH-PD approach, while the number particles has to be increased further in the standard PD solution. 
(a)

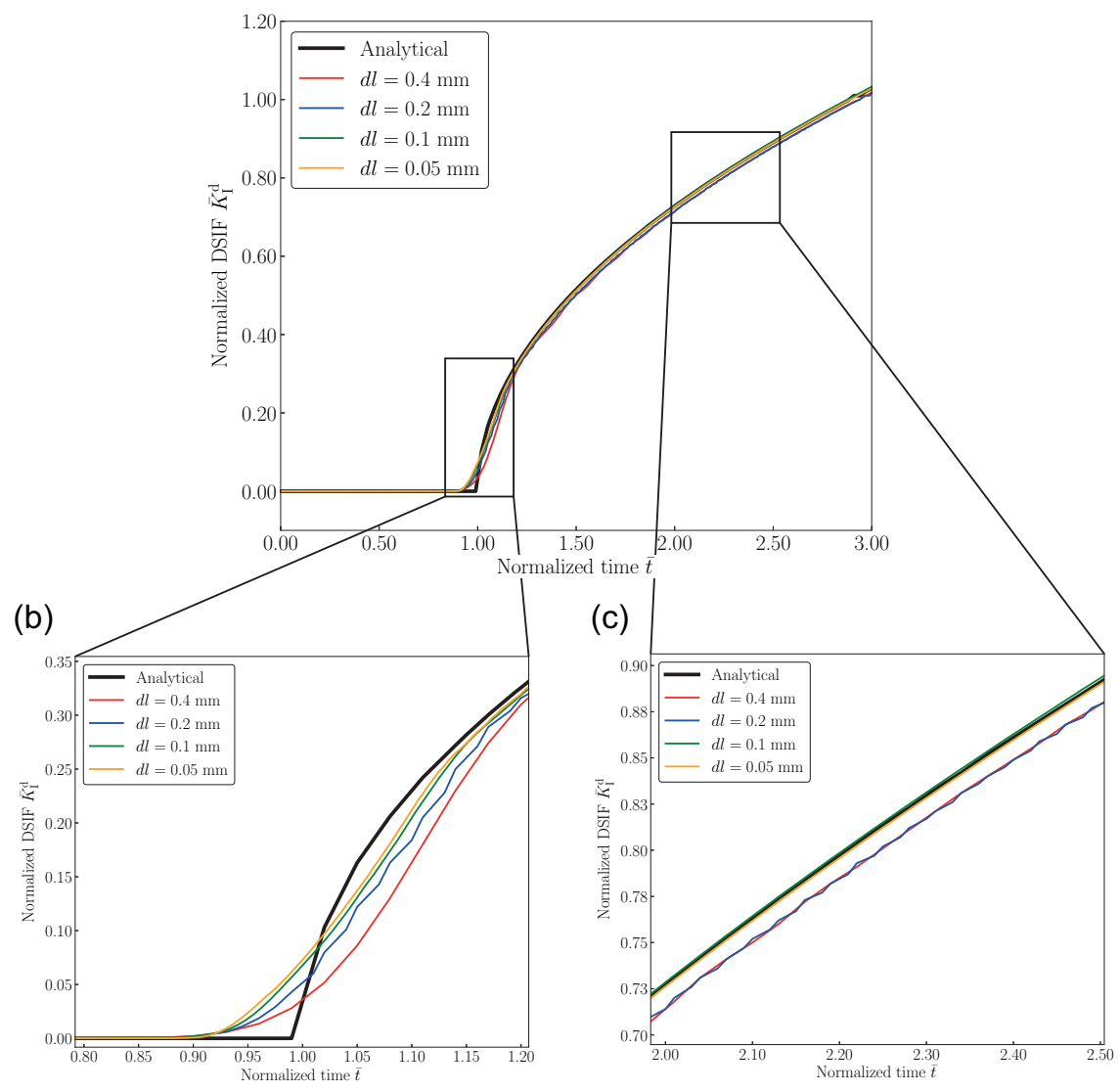

Fig. 20 Comparison of normalized DSIFs by the standard PD: (a) general trend of DSIFs, (b) close-up view between $\bar{t}=0.80$ and 1.20 , (c) close-up view between $\bar{t}=2.00$ and 2.50 .

\section{Conclusions}

The SVH-PD concept has been implemented for evaluating the DSIFs of stationary cracks. The undesired ghost force when the horizon size is changed has been addressed and the smoothing length concept was proposed to reduce the ghost force effect. It was clearly shown that the smoothing length concept significantly reduces the effect of ghost forces by distributing it through region, where the horizon size is varied gradually.

As for evaluating the DSIFs, the $J$-integral method has been employed. To evaluate displacement gradients in the $J$-integral formulation, MLS approximation was utilized. Path independence of the DSIFs obtained by the $J$-integral method has been verified. To improve the computational efficiency, the spatial resolution near the crack tip was gradually varied. This approach notably improved the $m$ convergence.

The DSIFs obtained by the standard PD and SVH-PD were compared with the reference analytical solution for a semi-infinite plate. Both results converged 
(a)

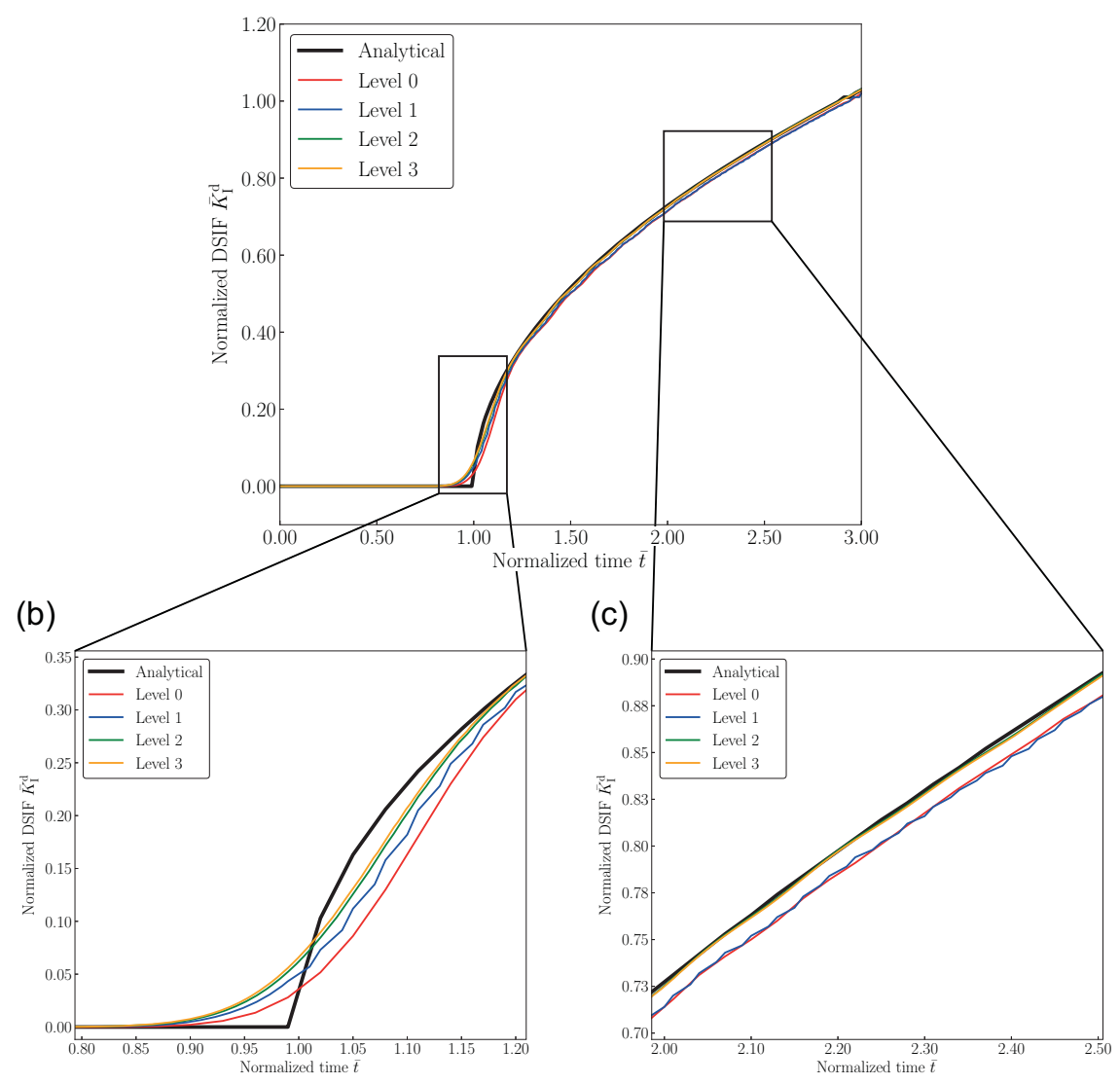

Fig. 21 Comparison of normalized DSIFs by VH-PD: (a) general trend of DSIFs, (b) close-up view between $\bar{t}=0.80$ and 1.20 , (c) close-up view between $\bar{t}=2.00$ and 2.50 .

to the analytical solution, however the computational efficiency of SVH-PD was significantly higher than that of the standard PD.

To sum up, the newly proposed smoothing length concept has reduced the ghost force effect and provided good accuracy while keeping the number of particles very low compared to the standard PD.

\section{Acknowledgement}

The present research was partially supported by the JSPS Grant-in-Aid for JSPS Fellows (19J14053) and Grants-in-Aid for Scientific Research (C)(18K04582). The authors gratefully acknowledged. 


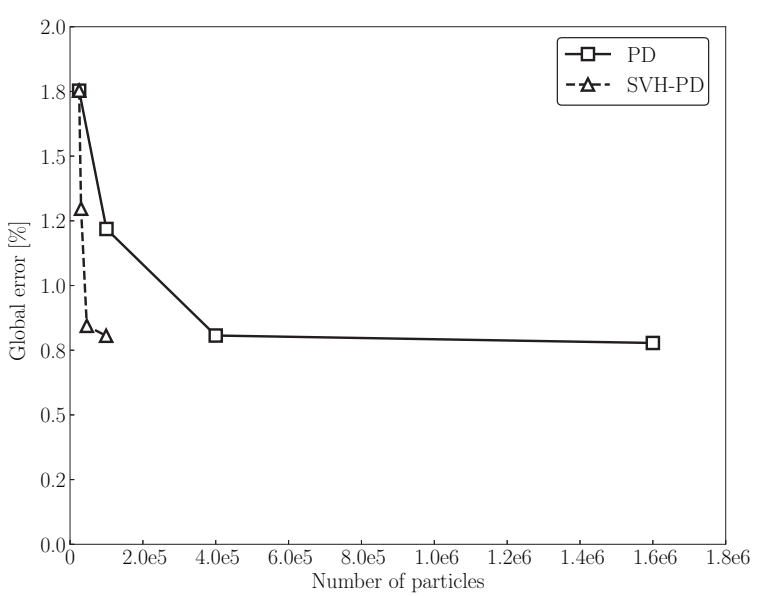

Fig. 22 Comparison of global error by the standard PD and SVH-PD.

\section{References}

1. Silling, S.A.: Reformulation of elasticity theory for discontinuities and long-range forces. J. Mech. Phys. Solid. 48, 175-209 (2000).

2. Ha, Y.D., Bobaru, F.: Studies of dynamic crack propagation and crack branching with peridynamics. Int. J. Fract. 162, 229-244 (2010).

3. Imachi, M., Tanaka, S.: Dynamics stress intensity factors evaluation employing ordinary state-based peridynamics. Trans. JSCES. 20160017 (2016). (in Japanese)

4. Imachi, M., Tanaka, S., Bui, T.Q.: Mixed-mode dynamic stress intensity factors evaluation using ordinary state-based peridynamics. Theor. Appl. Fract. Mech. 93, 97-104 (2018).

5. Imachi, M., Tanaka, S., Bui, T.Q., Oterkus, S., Oterkus, E.: A computational approach based on ordinary state-based peridynamics with new transition bond for dynamic fracture analysis. Eng. Fract. Mech. 206, 359-374 (2019).

6. Imachi, M., Tanaka, S., Ozdemir, M., Bui, T.Q., Oterkus, S., Oterkus, E.: Dynamic crack arrest analysis by ordinary state-based peridynamics. Int. J. Fract. 221, 155-169 (2020).

7. Ozdemir, M., Kefal, A., Imachi, M., Tanaka, S., Oterkus, E.: Dynamic fracture analysis of functionally graded materials using ordinary state-based peridynamics. Compos. Struct. 244, 112296 (2020).

8. Silling, S.A., Littlewood, D.J., Seleson, P.D.: Variable horizon in a peridynamic medium. Sandia Report. (2014).

9. Dipasquale, D., Zaccariotto, M., Galvanetto, U.: Crack propagation with adaptive grid refinement in 2D peridynamics. Int. J. Fract. 190, 1-22 (2014).

10. Song, Y., Yan, J., Li, S., Kang, Z.: Peridynamic modeling and simulation of ice craters by impact. Comput. Model. Eng. Sci. 121, 465-492 (2019).

11. Ren, H., Zhuang, X., Cai, Y., Rabczuk, T.: Dual-horizon peridynamics. Int. J. Numer Method Engrg, 108, 1451-1476 (2016).

12. Ren, H., Zhuang, X., Cai, Y., Rabczuk, T.: Dual-horizon peridynamics: A stable solution to varying horizons. Comput. Meth. Appl. Mech. Eng. 318, 762-782 (2017).

13. Ren, H., Zhuang, X., Rabczuk, T.: Implementation of GTN model in dual-horizon peridynamics. Procedia Engineer. 197, 224-232 (2017).

14. Rabczuk, T., Ren, H.: A peridynamics formulation for quasi-static fracture and contact in rock. Eng. Geol. 225, 42-48 (2017).

15. Tong, Q., Li, S.: Multiscale coupling of molecular dynamics and peridynamics. J. Mech. Phys. Solid. 95, 169-187 (2016).

16. Jenabidehkordi, A., Rabczuk, T.: The multi-horizon peridynamics. Comput. Model. Eng. Sci. 121, 493-500 (2019).

17. Nikpayam, J., Kouchakzadeh, M.A.: A variable horizon method for coupling meshfree peridynamics to FEM. Comput. Meth. Appl. Mech. Eng. 355, 308-322 (2019). 
18. Zhang, H., Li, H., Ye, H., Zheng, Y., Zhang, Y.: A coupling extended multiscale finite element and peridynamic method for modeling of crack propagation in solids. Acta Mech. 230, 3667-3692 (2019).

19. Khodabakhshi, P., Reddy, J.N., Srinivasa, A.: A nonlocal fracture criterion and its effect on the mesh dependency of GraFEA. Acta Mech. 230, 3593-3612 (2019).

20. Bessa, M.A., Foster, J.T., Belytschko, T., Liu, W.K.: A meshfree unification: reproducing kernel peridynamics. Comput. Mech. 53, 1251-1264 (2014).

21. Tanaka, S., Suzuki, H., Sadamoto, S., Okazawa, S., Yu, T.T., Bui, T.Q.: Accurate evaluation of mixed-mode intensity factors of cracked shear-deformable plates by an enriched meshfree Galerkin formulation. Arch. Appl. Mech. 87, 279-298 (2017).

22. Tanaka, S., Dai, M.J., Sadamoto, S., Yu, T.T., Bui, T.Q.: Stress resultant intensity factors evaluation of cracked folded structures by 6 DOFs flat shell meshfree modeling. Thin-Walled Struct. 144, 106285 (2019).

23. Dai M.J., Tanaka S., Sadamoto S., Yu T.T., Bui T.Q.: Advanced reproducing kernel meshfree modeling of cracked curved shells for mixed-mode stress resultant intensity factors. Eng. Fract. Mech. 233, 107012 (2020).

24. Ozdemir, M., Tanaka, S., Sadamoto, S., Yu, T.T., Bui, T.Q.: Numerical buckling analysis for flat and cylindrical shells including through crack employing effective reproducing kernel meshfree modeling. Eng. Anal. Bound. Elem. 97, 55-66 (2018).

25. Sadamoto, S., Ozdemir, M., Tanaka, S., Bui, T.Q., Okazawa, S.: Finite rotation meshfree formulation for geometrically nonlinear analysis of flat, curved and folded shells. Int. J. Non. Lin. Mech. 119, 103300 (2020).

26. Belytschko, T., Gu, L., Lu, Y.Y.: Fracture and crack growth by element free Galerkin methods. Modelling Simul. Mater. Sci. Eng. 2, 519-534 (1994).

27. Liu, W.K., Chen, Y.: Wavelet and multiple scale reproducing kernel methods. Int. J. Numer. Meth. Fluid. 21, 901-931 (1995).

28. Liu, W.K., Li, S., Belytschko, T.: Moving least-square reproducing kernel methods (I) methodology and convergence. Comput. Meth. Appl. Mech. Eng. 143, 113-154 (1997).

29. Silling, S.A., Epton, M., Weckner, O., Xu, J., Askari, E.: Peridynamic states and constitutive modeling. J. Elasticity. 88, 151-184 (2007).

30. Le, Q.V., Chan, W.K., Schwartz, J.: A two-dimensional ordinary, state-based peridynamic model for linearly elastic solids. Int. J. Numer. Meth. Eng. 98, 547-561 (2014).

31. Nikravesh, S., Gerstle, W.: Improved state-based peridynamic lattice model including elasticity, plasticity and damage. Comput. Model. Eng. Sci. 116, 323-347 (2018).

32. Bobaru, F., Yang, M., Alves, L.F., Silling, S.A., Askari, E., Xu, J.: Convergence, adaptive refinement, and scaling in 1D peridynamics. Int. J. Numer. Meth. Eng. 77, 852-877 (2009).

33. Cheng, Z., Wang, Z., Luo, Z.: Dynamic fracture analysis for shale material by peridynamic modelling. Comput. Model. Eng. Sci. 118, 509-527 (2019).

34. Rice, J.R.: A path independent integral and the approximate analysis of strain concentration by notches and cracks. J. Appl. Mech. 35, 379-386 (1968).

35. Li, F.Z., Shih, C.F., Needleman, A.: A comparison of methods for calculating energy release rates. Eng. Fract. Mech. 21, 405-421 (1985).

36. Shih, C.F., Moran, B., Nakamura, T.: Energy release rate along a three-dimensional crack front in a thermally stressed body. Int. J. Fract. 30, 79-102 (1986).

37. Nakamura, T., Shih, C.F., Freund, L.B.: Analysis of a dynamically loaded three-point-bend ductile fracture specimen. Eng. Fract. Mech. 25, 323-339 (1986).

38. Lancaster, P., Salkauskas, K.: Surfaces generated by moving least squares methods. Math. Comput. 37, 141-158 (1981).

39. Freund, L.B.: Dynamic fracture mechanics. Cambridge Monographs on Mechanics. (1990). 